\title{
LE EMANAZIONI DEL RADIO AUMBNTANO LA CONDUTTIVITA DELL'ACQUA
}

Nota del Dott. U. GRASSI ${ }^{1}$.

L'aumento di conducibilità che acquistano i liquidi sottoposti alle radiazioni più penetranti emesse dalle sostanze radioattive fu studiato dalla Curie ${ }^{2}$ ) con l' elettrometro e da Rubens e Kohlrausch ${ }^{8}$ ) con il metodo del ponte. Questi ultimi trovarono che un' acqua di conducibilita iniziale $1,1.10^{-6}$ $\mathbf{Q}^{-1}$ assume un potere conduttore crescente con il tempo in maniera più rapida quando è sottoposta alle radiazioni di quando non lo è; e precisamente l'aumento, non costante, è dell' ordine di grandezza di $0,025.10^{-6}$ in due giorni di esposizioni alle radiazioni e di $0,0050 \cdot 10^{-6}$ nel caso contrario. Questo interessante risultato si può spiegare ammettendo che i raggi emessi dal radio modifichino gli strati del liquido immediatamente colpiti e che poi, per movimenti convettivi facili ad immaginarsi, tale modificazione si diffonda nella massa del liquido stesso. Le emanazioni delle sostanze radioattive devono avere pure un notevole potere ionizzante, ed anche tenuto conto della loro minima solubilità nell' acqua, è presumibile che l'acqua cambi in qualche modo di conduttività facendovi gorgogliare un gas carico di tali emanazioni.

Feci a tal proposito alcune ricerche servendomi di un apparecchio in vetro di Jena della forma qui rappresentata (v. figura). L' apparecehio portava due elettrodi $E$ di platino terso, ed era immerso in un bagno ad olio di vaselina puro, immerso a sua volta in una grande vasca d'acqua delle dimensioni $50 \times 50 \times 40 \mathrm{~cm}$; la stanza da lavoro era cosi ben riparata, che la temperatura oscillava raramente di piu che un decimo di grado durante la gionnata. La lettura della temperatura del bagno di olio di vaselina era fatta con un

1) Rend, della R. Acc. dei Lincei, Voi, 14, Fasc. 5, $2^{4}$ sem., 1905.

2) Thesse, 1904.

3) luth. d. 1). Phys. Des. (5), n, 15, 19018. 
termometro in decimi, sul quale si potevano leggere comodamente a mezzo di un cannochiale anche i centesimi di grado;

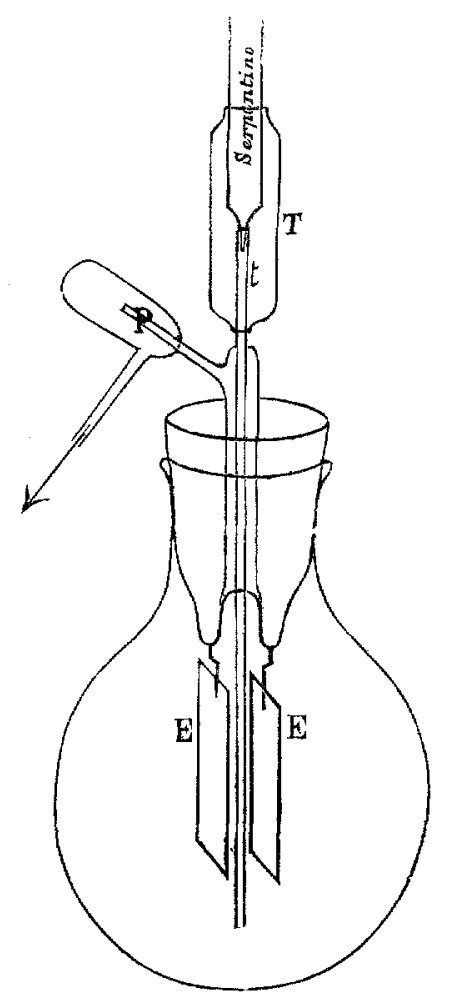

essa non oscillava mai di piu che $0,02^{\circ}$ in un intervallo di sei ore. Le misure di conducibilità furono eseguite con il metodo del ponte e del telefono; in un lato del ponte era situato l' apparecchio in vetro che serviva alle esperienze, in quello adiacente una resistenza metallica di $10000 \mathrm{Ohm}$; gli altri due lati erano costituiti da un reocordo di $20 \mathrm{Ohm}$, precedentemente calibrato, più 84 Ohm aggiunti ad esso; uno dei vertici del ponte era messo a terra, e la capacità elettrostatica del vaso elettrolitico veniva compensata con un condensatore di capacità variabile inserito parallelamente alla resistenza di 10000 Ohm. Con questo artifizio, cui si aggiunga 
la precauzione di tenere l' apparecchio induttore lontano dal resto del ponte di circa metri 1,5, l' aggiustamento del contatto scorrevole si potera fare con un errore $t 1$ decimillesimo del reocordo stesso; nei dati di resistenza la quarta cifra era perciò certamente esatta.

L' acqua adoperata era ottenuta per doppia distillazione con aggiunta di traccie di acido solforico e permanganato potassico, il serpentino del distillatore era di stagno e veniva ad infilarsi nel tubo $t$ dell' apparecchio elettrolitico; l' insieme veniva poi protetto ulteriormente da un manicotto $\mathrm{T}$ di vetro. Il tubo P, di efllusso, era pure protetto dall' aria nel modo indicato dalla figura. Del distillato si prendeva solo la porzione media e poichè si lasciava che per qualche tempo l'acqua che veniva dal serpentino circolasse nell' apparecchio, si raggiungeva un' energica lavatura dello stesso.

Le emanazioni venivano fatte gorgogliare attraverso all' acqua a mezzo di idrogeno, e siccome non era noto il comportamento dell' acqua sotto il gorgogliamento di questo gas, si presentava opportuno uno studio preliminare intorno ad esso. Era già noto che il gorgogliamento di aria pura aumen ta la resistenza dell' acqua distillata ${ }^{1}$ ), ma le osservazioni che ho avuto occasione di fare relativamente all' idrogeno, meritano di essere riferite. L' idrogeno era ottenuto dallo zinco ed acido solforico e veniva lavato attraverso $\mathrm{H}_{2} \mathrm{SO}_{4}, \mathrm{AgNO}_{3}, \mathrm{NaOH}$, $\mathrm{NaOH}$. L' acqua appena distillata presentava una conducibilità di $3,410 \times 10^{-7} \mathrm{Ohm}^{-1}$ a $22^{0}, 55$ e di $3,493 \times 10^{-7} \mathrm{Ohm}^{-1}$ a $22^{0}, 93$. Il gorgogliamento dell' idrogeno produceva un notevole aumento della resistenza, tanto che dopo tre giorni circa di lento e regolare gorgogliamento l' acqua presentava una conducibilita due volte appena superiore a quella della migliore acqua ottenuta da Kohlrausch, ossia una conducibilità di $1,016.10^{-7} \mathrm{Ohm}{ }^{4}$ a $23^{0}, 09^{2}$ ); e continuando il gorgogliamento, si manteneva costantemente ad un tale valore. Appena si interrompe il gorgogliamento, la conducibilità aumenta con una

1) Kohlrausch. Wied. Ann. 44, 585, 1891 ; Pfeiffer, Wied. Antı. 37, 539, 1889.

2) L' arqua ottenuta du $K_{0}$ hlrausch alla modesina temperatura possedera fa conducibilitá di $0,492.10^{-i}$. 
certa rapidità, peraltro tornando poi a metterlo in azione si ritorna ai valori primitivi con grandissima rapidita; si aggiunga a ciò che anche lo scuotere l' apparecchio cagiona un aumento della resistenza del liquido: questo mi induce a credere che l'aumento di resistenza prodotto con il trattamento descritto non sia esclusivamente da imputarsi ad uno spostamento di tracce minime di anidride carbonica sciolta nell' acqua; non credo poi che con il gorgogliamento si fosse finora raggiunto il limite al quale io ho potuto arrivare.

Le emanazioni si ricavano da due milligrammi di bromuro di radio dato per puro dalla Chinin-Fabrik di Braunschweig. Essi erano posti in una piccola boccia di Woolf attraverso la quale passava l'idrogeno purificato come precedentemente; prima di mandare le emanazioni nel vaso elettrolitico, si aveva cura di lasciare che l'idrogeno circolasse per qualche tempo; poi si chiudeva il tubo di efflussis con un rubinetto smerigliato e si lasciava riposare l' apparecchio; in tal maniera l' idrogeno che riempiva la boccia di Woolf si caricava di emanazioni; per effetio del gorgogliamento delle emanazioni la conducibilità aumentava rapidamente, come risulta dal seguente prospetto:

\begin{tabular}{|c|c|c|c|c|c|c|c|c|c|}
\hline \multirow[b]{2}{*}{ T'empo } & \multicolumn{9}{|c|}{ Temperatura $22^{\circ}, 9: \pm 0,01$} \\
\hline & 0 & $30^{17}$ & $66^{111}$ & $65^{111}$ & $70^{\mathrm{In}}$ & $75^{\mathrm{II}}$ & $8 i^{111}$ & $95^{\mathrm{m}}$ & $110^{\mathrm{m}}$ \\
\hline Condue. & 1,016 & 1,180 & 1,359 & 1,495 & 1,846 & 1,982 & 2,060 & 2,110 & $2,223 \times 10^{-7} \mathrm{Ohm}^{-1}$ \\
\hline Temp & $140 \mathrm{~m}$ & 17 & & 29 & & $365^{\mathrm{m}}$ & 50 & & $960^{\mathrm{m}}$ \\
\hline Conduc. & 2,297 & 2,4 & & 2,6 & & 2,765 & 2,7 & & $3,056 \times 10^{-7} 0 \mathrm{~lm}^{-1}$ \\
\hline
\end{tabular}

L'aumento di conducibilità non è peraltro costante, esso sembra dipendere dal tempo per cui l'idrogeno destinato a caricarsi di emanazioni è rimasto a contatto con il radio; cosi, ad esempio, avendolo lasciato per circa due settimane partendo da un' acqua della conducibilità $3,010.10^{-7}$ ottenni un' acqua della conducibilità di $1,090.10^{-6} \mathrm{Ohm}^{-1}$. L' aumento cospicuo di conducibilitż mi fece sulle prime sospettare che del bromo si liberasse per avventura dal bromuro di radio; imaginando però che il bromo entrasse in soluzione allo stato di acido bromidrico, si sarebbe dovuto avere uno sviluppo di almeno 
$0,8 \cdot 10^{-4}$ gr. circa di bromo ${ }^{1}$ ); quantita troppo notevole, data la esigua quantità del compcsto; ina ho eseguita una prova che mi sembra anche più decisiva, inquantochè ho lavato le emanazioni stesse attraverso nitrato di argento e soda concentrata ed ho ottenuto il seguente andamento $\left.{ }^{2}\right)$ :

$$
\begin{array}{ccccccc}
\text { Tempo } & 0 & 80^{\mathrm{m}} & 140^{1 \mathrm{~h}} & 210^{\mathrm{m}} & 280 \mathrm{~m} & 440 \mathrm{~m} \\
\text { Conduc. } & 1,016 & 1,138 & 1,167 & 1,189 & 1,209 & 1,288 \times 10^{-7} \mathrm{Ohm}^{-1}
\end{array}
$$

È mio proposito di proseguire queste ricerche preliminari allo scopo di vedere se le emanazioni hanno pure influenza sulla conducibilità degli elettroliti sciolti nell' acqua. Si dorrà perciò scegliere qualche elettrolito assai debole (come l'anilina ed il fenolo); ho ancora intenzione di indagare il comportamento delle emanazioni verso solventi diversi dall' acqua, e con gas diversi dall' idrogeno, ed in particolare il loro comportamento verso l'acetone e l'acetilene che è in esso solubilissimo. Queste ulteriori ricerche potranno rivelare in modo deciso quale contributo portino al fenomeno osservato, i gas che sviluppano le sostanze radifere specialmente in presenza di umidità.

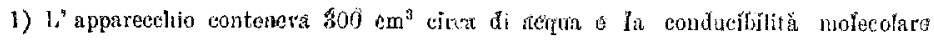
Jell' acido bromidrico è di cirea $386 \mathrm{0}_{\mathrm{h}} \mathrm{m}^{-1}$.

2) Non è presumibile che le acque di lavagyio assorbiscano una quantitì cosi cospicua di emanazioni quale risulterebbe dai numeri del prospetto confrontati con i precedenti : si dove peraltro notare che l'idkogeno riduce il nitrato d'arsento, e l'argento metallico così generato può benissimn assorbire una farto notevole di emanazioni rimaneudo a sua volta radioativo. 